\title{
RESPUESTA DE UNA CUENCA DE CABECERA DURANTE EVENTOS DE CRECIDA (AIXOLA, PAÍS VASCO)
}

\author{
A. ZABALETA \& I. ANTIGÜEDAD \\ Grupo de Hidrogeología y Medio Ambiente, Departamento de Geodinámica, Universidad del País Vasco- \\ Euskal Herriko Unibertsitatea, 48940 Leioa, Bizkaia, País Vasco. \\ E-mail: ane.zabaleta@ehu.es \\ ABSTRACT.- Turbidity ( $T, F N U)$, discharge $(Q, l / s)$ and precipitation $(P, \mathrm{~mm})$ \\ are being continuously monitored in the gauging station located at the outlet of \\ Aixola catchment (Gipuzkoa, $5 \mathrm{~km}^{2}$ ) since October 2003. Several data sets recorded \\ during flood events occurred between 2003 and 2005 were used to estimate contin- \\ uous suspended sediment concentration (SSC). Electrical conductivity was also \\ measured in samples taken during runoff events. Several event (discharge, precipi- \\ tation and suspended sediment concentration) and pre-event (discharge and precip- \\ itation) factors are calculated for all the events registered. With them a correlation \\ matrix was developed. Good correlation between precipitation $(P)$, discharge $(Q)$ and \\ suspended sediment (SS) variables is found in general but pre-event conditions don't \\ show correlation with event parameters. Analysis of SSC-discharge evolutions \\ through the events showed that four different kinds of hysteretic loops can be \\ observed in Aixola. Hysteretic loops have also been related to event and pre-event \\ factors. Evolution of electrical conductivity of waters during events shows that the \\ catchment has a considerable regulation capacity.
}

Keywords: Discharge; suspended sediment concentration; electrical conductivity; single flood events; hysteretic loops; headwater catchment.

RESUMEN.- La turbidez ( $T, F N U)$, el caudal $(Q, l / s)$ y la precipitación $(P$, mm) se están midiendo en continuo en la estación de aforo situada en la salida de la cuenca de Aixola (Gipuzkoa, $5 \mathrm{~km}^{2}$ ) desde octubre 2003. Se han utilizado los datos recogidos en los eventos ocurridos entre octubre 2003 y octubre 2005 para establecer la relación turbidez-concentración de sedimentos en suspensión (CSS), y así, estimar las series continuas de CSS. Además, también se ha medido la conductividad eléctrica en muestras tomadas durante las crecidas. Por otro lado, se cuantificaron varios parámetros del evento ( $P, Q$ y CSS) y anteriores al mismo ( $Q$ y $P$ ) para todos los eventos registrados. Se observa un nivel significativo de correlación entre las varia- 
bles de precipitación, caudal y sedimentos en suspensión. Sin embargo, las condiciones anteriores al evento no se correlacionan bien con los parámetros calculados para el mismo. En el análisis de la relación entre la concentración de sedimentos en suspensión y el caudal durante los eventos se han observado cuatro tipos diferentes de bucles de histéresis relacionados con diferentes condiciones antecedentes al evento y del propio evento. La evolución de la conductividad eléctrica durante los eventos de crecida indica que la cuenca posee una capacidad de regulación considerable.

Palabras clave: Caudal; concentración de sedimentos en suspensión; conductividad eléctrica; eventos de crecida; bucles de histéresis; cuenca de cabecera.

\section{Introducción}

Los ríos constituyen agentes de erosión y transporte, y forman el mayor vínculo entre los sistemas marino y terrestre (KNIGHTON, 1998), transfiriendo más de $35 \cdot 10^{3} \mathrm{~km}^{3}$ de agua y $20 \cdot 10^{9} \mathrm{t}$ de sólidos disueltos y en suspensión todos los años (FARNSWORTH \& MILLIMAN, 2003). Por lo tanto, son los responsables de llevar continuamente el agua y los productos del lavado y la erosión generados en el continente desde las cuencas de cabecera hasta las zonas de deposición. Además, las principales fuentes del agua y de los sedimentos que llegan a los océanos son las cuencas de cabecera (SCHUMM, 1977). En general, la inmensa mayoría del material sólido es transportado por el caudal en forma de sedimentos en suspensión que según las estimaciones suponía entre 15 y $18 \cdot 10^{9}$ t/año (HOLEMAN, 1967, MILLIMAN \& MEADE, 1983, MILLIMAN \& SYVITSKI, 1992), de las cuales sólo el $40 \%$ eran exportadas por las grandes cuencas de drenaje $\left(>10^{4} \mathrm{~km}^{2}\right)$ MILLIMAN \& SYVITSKI (1992). Algunas investigaciones más recientes (FARNSWORTH \& MILLIMAN, 2003) corroboran, además de estos últimos datos, la importancia de las pequeñas cuencas de montaña en el cómputo global de exportación de sedimentos en suspensión.

Además, el sedimento fino ha sido identificado como un importante vector para el transporte de nutrientes (WALLING et al., 2001) y contaminantes como los metales pesados (ANKERS et al., 2003) y los microorganismos (HOUSE et al., 1997). Sin embargo, el sedimento en suspensión es importante en sí mismo, ya que su presencia o ausencia ejerce un importante control en los procesos geomorfológicos y biológicos de ríos y estuarios (WASS \& LEEKS, 1999). Quizás por ello, en las últimas décadas ha crecido de manera importante el interés existente sobre la dinámica de los sedimentos en suspensión. 
Uno de los pioneros en evaluar la carga en suspensión en ríos vascos fue Felix M. Ugarte motivado, sobre todo, por la destrucción del suelo debido a la acción humana (actividad forestal, obras públicas...). Entre otros, trató de medir las consecuencias de una agresiva actividad forestal en una ladera de pendiente media acusada $(60 \%)$ en la que se midieron valores de concentración de sedimentos en suspensión de hasta $2500 \mathrm{mg} / 1$ y pérdidas de suelo de hasta 2600 t/ha·año (de PABLO et al., 1991). También analizó la carga en suspensión del río Oñati en relación con su dinámica fluvial (BARTUREN \& UGARTE, 1988), concluyendo la escasa relación existente entre la carga y el caudal y la importancia de las situaciones climáticas (intensas precipitaciones, deshielos) en los valores obtenidos de carga en suspensión. Más adelante, se encuentran los trabajos realizados por Edeso (Ej: EDESO, 1997) en pequeñas parcelas o laderas bajo actividad forestal en las que se ha tratado de medir sus tasas de erosión. También se han realizado algunos estudios a una escala mucho mayor, como los trabajos de URIARTE (1998) en los ríos principales de Gipuzkoa, con datos de exportación de sedimentos, para cuencas de entre 40 y $780 \mathrm{~km}^{2}$, de entre 45 y $260 \mathrm{t} / \mathrm{km}^{2} \cdot$ año, obtenidos a partir de medidas puntuales, o los realizados por MANEUX et al. (1999) en el Urdazuri (Nivelle) donde se estimaron tasas de exportación de sedimentos en suspensión que varían entre 60 y $84 \mathrm{t} / \mathrm{km}^{2}$ ·año. Además, estos autores quisieron subrayar la importancia de los pequeños ríos de montaña en la exportación total de sedimentos al Golfo de Bizkaia, ya que sus tasas de aportación suponen más del $50 \%$ del sedimento que llega a las costas.

Los trabajos realizados en los últimos años en este sentido en la cuenca de Aixola $\left(4.8 \mathrm{~km}^{2}\right)$ (ZABALETA et al., 2007, ZABALETA, 2008) pretenden representar un avance en el conocimiento de la respuesta hidrosedimentaria de las pequeñas cuencas de cabecera de la provincia de Gipuzkoa y, por extensión, de otras con características climáticas y geomorfológicas similares. Así, el objetivo general del presente trabajo es aportar una primera aproximación a los factores que puedan resultar necesarios para un mejor conocimiento de la dinámica hidrológica y sedimentaria de una pequeña cuenca de cabecera, en este caso Aixola, a través de la caracterización de las relaciones entre el caudal y los sedimentos en suspensión durante los eventos de crecida, su relación con las características de las mismas y del estudio de la evolución de la conductividad eléctrica durante los eventos.

\section{2. Área de estudio}

El río Aixola se ubica al oeste de la provincia de Gipuzkoa (Fig. 1), en la cuenca media del río Deba, entre las localidades de Elgeta y Ermua. En este 
trabajo se ha considerado la cuenca que drena dicho río hasta la estación de aforos del mismo nombre, ubicada aguas arriba del embalse de Aixola, con una capacidad de $2.73 \mathrm{hm}^{3}$ y cuyas aguas son utilizadas para abastecer de agua potable al municipio de Eibar. El área de estudio consta de $4.8 \mathrm{~km}^{2} \mathrm{y}$ presenta un desnivel de $410 \mathrm{~m}$, desde los $340 \mathrm{~m}$ de altura de la estación de aforos hasta los $750 \mathrm{~m}$ que tiene el monte Intxorta, ubicado al sur de la cuenca. Se trata de una zona con pendientes que en general no sobrepasan el $30 \%$.

La cuenca de Aixola representa un caso extremo de cuenca reforestada, donde la vegetación autóctona está restringida a pequeños enclaves mientras que las especies forestales introducidas ocupan prácticamente todo el territorio, de modo que la especie reina es el Pinus radiata, aproximadamente un $80 \%$ de la cuenca está cubierta de este pino. En el resto de la cuenca se encuentran, por orden de importancia, las praderas de gramíneas, otras especies introducidas, como el Larix decidua y el Abies alba, y, por último, un reducto de bosque mixto de hayas (Fagus sylvatica) y robles (Quercus robur).

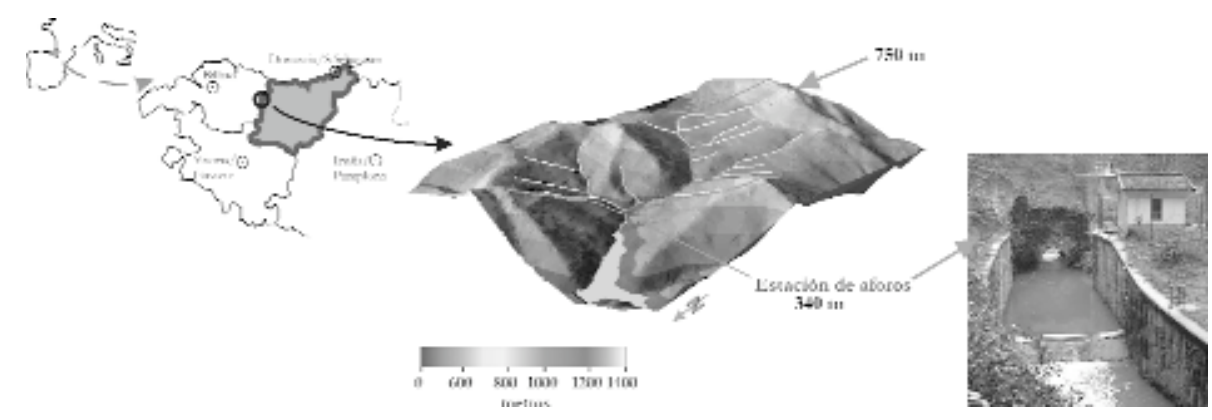

Figura 1. Localización y representación del modelo digital del terreno de la cuenca de Aixola. Figure 1. Location and digital terrain model of Aixola catchment.

La mayor parte de los materiales que afloran en la cuenca (el 94\%) pertenecen al Flysch detrítico calcáreo (Santoniense-Maastrichtiense Medio, Cretácico Superior). En general, la serie está formada por una alternancia de margas y calizas arenosas en bancos centi-decimétricos (entre 5 y 20 centímetros generalmente). Todo el conjunto constituye una serie turbidítica con algunas variaciones locales más o menos importantes. Al sur de la cuenca existen pequeños afloramientos de la formación calcárea del Cretácico Superior que pueden encontrarse intercalados en el complejo volcánico en el que se engloban "sills" básicos y rocas volcanoclásticas. Los principales suelos presentes en la cuenca de Aixola son cambisoles y regosoles (F.A.O, 1991) relativamente profundos. 
La precipitación media anual calculada a partir de los datos medidos en la estación de aforos de Aixola desde el año 1986-1987 hasta el 2005-2006 es de $1480 \mathrm{~mm} /$ año, con totales de 1450 y $1375 \mathrm{~mm}$ / año durante los años 200304 y 2004-05. En cuanto a las salidas de la cuenca, la escorrentía media registrada en la estación de aforos durante los 21 años de control (1986-2006) es de $600 \mathrm{~mm}$, siendo 667 y $800 \mathrm{~mm}$ para los años 2003-04 y 2004-05, respectivamente. Mientras el caudal circulante por la estación de aforos entre eventos de crecida tiene una conductividad eléctrica de unos $370 \mu \mathrm{S} / \mathrm{cm}$, ésta muestra valores considerablemente menores, por debajo incluso de los 200 $\mu \mathrm{S} / \mathrm{cm}$, durante los eventos de crecida. Durante el periodo 2003-2008, la tasa media de exportación de sedimentos en suspensión cuantificada, a partir del control continuo, para la cuenca ha sido de $36 \mathrm{t} / \mathrm{km}^{2}$, con concentraciones medias de $128 \mathrm{mg} / 1$ durante los eventos (alrededor de 200 en 5 años), y exportándose la mayor parte del sedimento entre noviembre y abril (ZABALETA, 2008).

\section{Aspectos metodológicos}

A la salida de la cuenca se localiza una estación de aforo perteneciente a la red foronómica de la Diputación Foral de Gipuzkoa. La instrumentación previamente existente en esa estación, en la que se miden precipitaciones y caudal, entre otros, se completó en octubre de 2003 con un fotómetro Solitax ${ }^{\circledR}$ para medir la turbidez y un tomamuestras portátil automático Sigma 900, ambos de Dr. Lange, con la finalidad de obtener información sobre los procesos de exportación de sedimentos en suspensión. La turbidez se mide en el Solitax ${ }^{\circledR}$ mediante la dispersión de haces de luz infrarroja dual (dispersión y retrodispersión) con un rango de medida de 0 a 1000 FNU. Este sensor óptico, utiliza la evaluación paralela de diferentes haces de luz para dar una medición de la turbidez independiente del color. Al igual que en el caso del caudal y la precipitación, se guarda un dato de turbidez cada diez minutos. El muestreador automático se programó para coger muestras, unos $600 \mathrm{ml}$ en cada botella, cuando la lámina de agua en la estación sobrepasa un nivel preestablecido que varía, según la época del año, en torno a una media de $17 \mathrm{~cm}$ $\left(248 \mathrm{l} / \mathrm{s}, 51.66 \mathrm{l} / \mathrm{s} \cdot \mathrm{km}^{2}\right)$.

Los turbidímetros se pueden utilizar para estimar el flujo continuo de sedimentos en suspensión (GIPPEL, 1989; BRASINGTON \& RICHARDS, 2000), sin embargo, para establecer una relación veraz entre la turbidez (parámetro de medida) y la concentración de sedimentos en suspensión (parámetro de interés) se requiere un programa de muestreo de sedimentos muy exhaustivo con una extensiva recogida de datos a intervalos de tiempo 
lo más cortos posibles (LAIGNEL et al., 2006) y una serie de datos que se pueda usar para interpolar más que para extrapolar (SUN et al., 2001). Por ello se establecieron programas de muestreo adecuados a las características de las crecidas en las diferentes épocas del año, más duraderas y con varios picos en otoño-invierno y más cortas e intensas en primavera-verano (ZABALETA, 2008). En las muestras recogidas por el tomamuestras se ha medido directamente en el campo la conductividad eléctrica, con un conductivímetro Crison 524. Después, todas las muestras recogidas en la estación de aforo son llevadas al laboratorio para medir la turbidez de las mismas, con un turbidímetro WTW Turb 555 IR que tiene un rango mayor que el turbidímetro de campo, de 0 - 10000 NTU, y cuantificar la concentración de sedimentos en suspensión que contienen filtrando las muestras a través de filtros de nylon Millipore de poros de $45 \mu \mathrm{m}$ de diámetro. De este modo es posible establecer la relación entre la turbidez y la concentración de sedimentos en suspensión y derivar series continuas de concentración de sedimentos a partir de la serie de turbidez (LEWIS, 1996; ZABALETA, 2008).

Teniendo en cuenta las lecturas diezminutales de los diferentes parámetros se caracterizaron 76 eventos de crecida registrados en Aixola (2003-2005) mediante cuatro grupos de parámetros (ZABALETA et al., 2007): condiciones antecedentes al evento, precipitación que causa el evento, caudal durante el evento y sedimentos en suspensión durante el evento. Las condiciones antecedentes al evento han sido descritas mediante la precipitación acumulada durante una hora antes del evento $(\mathrm{aP} 1, \mathrm{~mm})$, durante uno, siete y veintiún días anteriores al evento $(\mathrm{aP} 1 \mathrm{~d}, \mathrm{~mm}$; $\mathrm{aP7d}, \mathrm{mm}$; aP21d, mm), y el caudal medio registrado en la estación de aforos durante el día (o las 24 horas) anterior al evento (aQ1d, 1/s). Este último parámetro, aQ1d, a falta de datos que indiquen la humedad del suelo directamente, permite tener una idea de las condiciones de humedad de la cuenca antes del evento. La precipitación causante de los eventos ha sido caracterizada mediante la precipitación total registrada $(\mathrm{Pt}, \mathrm{mm})$, la intensidad media de la precipitación durante el evento lluvioso (IP, $\mathrm{mm} / \mathrm{h}$ ) y la intensidad máxima de la precipitación (IPmax, $\mathrm{mm} / 10 \mathrm{~min}$ ). IPmax es la precipitación máxima registrada en los datos diezminutales disponibles. Los datos de precipitación son los registrados en la estación de aforos situada en la parte baja de la cuenca - no existe ningún otro dispositivo de medida en la cuenca.

El caudal durante el evento se ha expresado utilizando el volumen de agua específico total del evento de escorrentía $(\mathrm{Qt}, \mathrm{mm})$, el caudal medio (Qmed, 1/s) y el máximo (Qmax, 1/s), y la relación entre este caudal máximo y el caudal inicial anterior al evento (Qmax/Qb).

La exportación de sedimentos en suspensión se ha explicado con la media de los datos de concentración de sedimentos en suspensión derivados cada 
diez minutos durante el evento (CSSmed, $\mathrm{mg} / \mathrm{l}$ ), la concentración máxima del evento (CSSmax, mg/l) y la exportación total de sedimentos en suspensión del evento (SSt, kg). Una vez definidos los conjuntos de parámetros se ha realizado una matriz de correlación de Pearson y un Análisis de Componentes Principales con rotación Varimax con el fin de conocer los parámetros que puedan tener una mayor influencia sobre la respuesta de los sedimentos en suspensión.

La conductividad eléctrica no se pudo incluir en este análisis estadístico debido a que no se dispone de medidas continuas, sin embargo, también se ha analizado la evolución de la conductividad eléctrica durante los eventos de crecida y su relación con el caudal y la concentración de sedimentos en suspensión.

\section{Resultados}

Con los 76 eventos caracterizados se realizó una matriz de correlaciones con el fin de identificar las posibles relaciones entre los parámetros calculados para las crecidas (Tabla 1). De esos 76 eventos el 38\% ocurrió en otoño, el 25\% en invierno, otro $25 \%$ en primavera y el $12 \%$ restante en verano. La matriz de correlación realizada con los parámetros definidos muestra que, por un lado, la precipitación total $(\mathrm{Pt})$ durante el evento está bien correlacionada con todos los parámetros de caudal, Qmed, Qt y Qmax y también con Qmax/Qb, aunque este último parámetro, $Q \max / \mathrm{Qb}$, se correlaciona mejor con la intensidad máxima de la precipitación (IPmax). Pt también está fuertemente relacionado con la exportación total de sedimentos del evento $\left(R^{2}=0.58\right)$, mientras que CSSmed y CSSmax se correlacionan mucho mejor con la intensidad máxima de la precipitación $\left(\mathrm{R}^{2}=0.72,0.55\right.$, respectivamente). Por otro lado, los parámetros relacionados con el caudal que tienen un mayor control en la exportación y la concentración de sedimentos en suspensión son Qmax y el parámetro Qmax/Qb. De acuerdo con SEEGER et al. (2004), otro de los factores importantes en la exportación de sedimentos en suspensión son las condiciones antecedentes, aunque, en el caso de Aixola, éstas no parecen tener relación significativa con el caudal o con los sedimentos en suspensión generados durante el evento.

Un análisis factorial (Fig. 2) de los parámetros citados agrupa en el factor I a los parámetros IP, IPmax, CSSmed, CSSmax y Qmax/Qb, explicando el $29 \%$ de la varianza. En el factor II se agrupan Pt, Qt y Qmed explicando el $23 \%$ de la varianza. En el plano factorial I-II, se observa bien que la exportación total de sedimentos en suspensión (SSt) muestra una buena relación con los parámetros de ambos factores aunque preferentemente con 
Tabla 1. Matriz de correlaciones de Pearson entre los parámetros calculados para todos los eventos de Aixola ocurridos entre 2003 y 2005 ( $n=76$ ). La correlación es significativa al nivel 0.01 para los números en negrita y al 0.05 para cursivas.

Table 1. Pearson correlation matrix between parameters calculated for all the events registered in Aixola catchment between 2003 and 2005 (n=76). Correlation is significant at the 0.01 level for bold numbers and 0.05 for italics.

\begin{tabular}{|c|c|c|c|c|c|c|c|c|c|c|c|c|c|c|c|}
\hline & $\mathrm{I}$ & $I_{p}$ & Ipenas & Al' & alind & alind & IVtad & 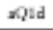 & Qanod & $Q x$ & Qmax & Gmax/Qb & CSSmod & CSOmaI & SSt \\
\hline $\mathrm{P}:$ & 100 & & & & & & & & & & & & & & \\
\hline W. & 0.15 & 20 & & & & & & & & & & & & & \\
\hline Ipmex & 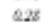 & 0.52 & 100 & & & & & & & & & & & & \\
\hline PI & $0.07 ?$ & -0.12 & -0.10 & 1.00 & & & & & & & & & & & \\
\hline aplel & กต & 2,16 & ans & 0.40 & $1 \mathrm{~m}$ & & & & & & & & & & \\
\hline arlid & turs & $A z$ & NOS & 0.52 & $\alpha z$ & 100 & & & & & & & & & \\
\hline IP21d & .0 .15 & 33.9. & -4.34 & 0.30 & 024 & 0.64 & 1.90 & & & & & & & & \\
\hline QU & -19 & 4 & as & וצטם & a.r. & דים & uns & Iנ: & & & & & & & \\
\hline Qumerat & 0.50 & .010 & -0.3 & 0.38 & 0.34 & 0.51 & 0.40 & 0.67 & 1.00 & & & & & & \\
\hline$\phi t$ & 0.60 & 0.15 & 0.5 & 0.25 & 0.18 & 0.33 & 0.27 & 0.45 & 0.58 & 100 & & & & & \\
\hline $\mathrm{Q}=\mathrm{ms}$ & ash & 4. .71 & 0.43 & 1030 & 0.14 & $n: X$ & wh & a.x & 0.T. K & uex & 1.101 & & & & \\
\hline Ueass/Q & 0.54 & 0.54 & 0.70 & -0.12 & -0.14 & $\Delta 21$ & -4.37 & $a .28$ & 0.11 & 0.13 & 0.65 & 1.00 & & & \\
\hline CStimad & 0.34 & ans & 0.72 & two & tiun & t11s & -4.15 & e.1.1? & แาง & ints & aA1 & and & 1.60 & & \\
\hline CSSanes & 0.33 & 0.20 & 0.55 & $a 06$ & S.11 & 0.00 & 0.28 & 0.17 & 0.07 & 0.03 & 0.57 & 0.85 & 0.92 & 1.00 & \\
\hline $\mathrm{ss}$ & 4.59 & 027 & 0.77 & nox & $\mathrm{am}$ & $n \infty$ & 611 & non & R49 & 4.51 & 0.55 & $n \pi r_{i}$ & 0.72 & 0.80 & 100 \\
\hline
\end{tabular}

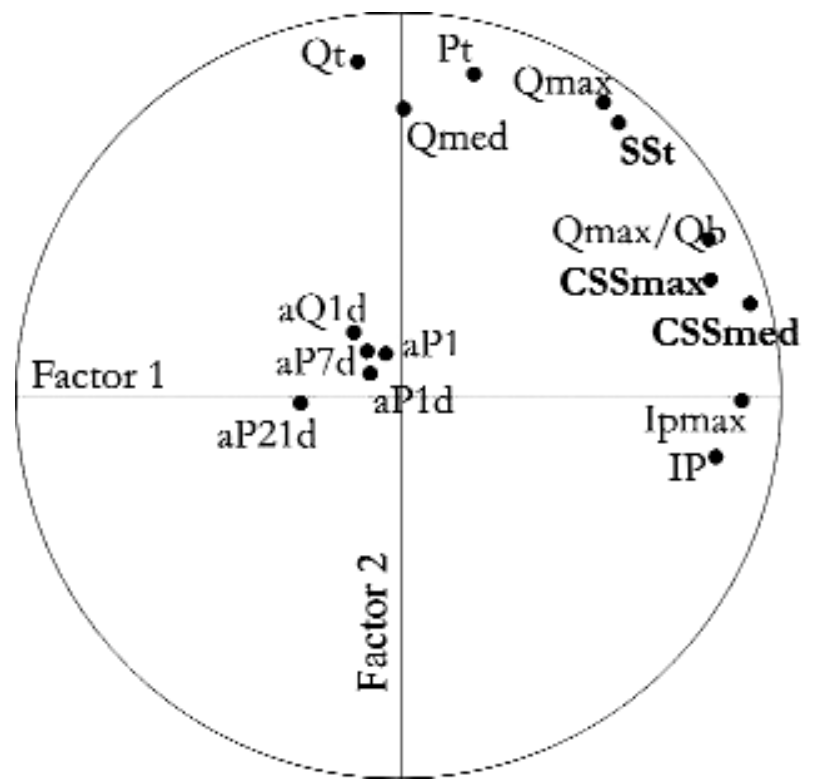

Figura 2. Localización de los parámetros incluidos en las matrices de correlación en el plano factorial I (eje horizontal) - II (eje vertical) del análisis de componentes principales.

Figure 2. Location of parameters included in the correlation matrixes in the I (in the horizontal axis)-II (in the vertical axis) factorial plane of the principal component analysis. 
los del factor I, y no presenta relación significativa con las condiciones anteriores al evento.

Además de estudiar la relación entre los parámetros hidrológicos y de transporte de sedimentos del evento, también se ha analizado la relación entre la concentración de sedimentos en suspensión y el caudal a lo largo del evento. La mayoría de las veces esta relación no es homogénea durante los eventos produciendo bucles de histéresis (WILLIAMS, 1989; LLORENS et al., 1997; SICHINGABULA, 1998; LENZI y MARCHI, 2000; PICOUET et al., 2001; ALEXANDROV et al., 2003; SEEGER et al., 2004). Así, de los 76 eventos analizados, 22 presentan una relación lineal entre la concentración y el caudal, 18 un bucle en sentido horario, 26 un bucle anti-horario, 1 en forma de ocho y 9 no han sido definidos.

Un análisis factorial (Fig. 3) en el que se han tenido en cuenta la precipitación (Pt e IPmax), el caudal (Qmed), la exportación de sedimentos (SSt) y las condiciones antecedentes (aP12, la precipitación acumulada en las 12 horas anteriores al comienzo del evento) muestra que cada tipo de bucle de histéresis se relaciona con unas condiciones hidrológicas diferentes. Los dos componentes principales creados explican el $71 \%$ de la varianza, con la precipitación total, el caudal medio y la exportación de sedimentos en la parte positiva del primer factor (explicando el $40 \%$ de la varianza) y en el factor dos (explicando el $31 \%$ de la varianza) la intensidad máxima de la precipitación en el lado negativo y la precipitación de las 12 horas previas en el positivo.

La figura 3 muestra la posición de los distintos tipos de eventos en el plano factorial. Los eventos horarios se ubican en la parte positiva del primer y el segundo factor, por lo que se pueden describir como eventos con grandes precipitaciones y caudales y mucha exportación de sedimentos en suspensión, y además que se dan en condiciones de abundantes precipitaciones antecedentes. Este tipo de eventos se observan, sobre todo, entre octubre y abril. En estos casos el pico de concentración de sedimentos llega antes a la estación de aforos que el pico de caudal y, por lo tanto, el valor de la concentración en la rama ascendente del hidrograma es mayor que el de la rama descendente. Entre las crecidas definidas por un solo pico en el hidrograma se ha seleccionado la del 16 de noviembre de 2003 a modo de ejemplo (Fig. 4). En dicha crecida se registró una precipitación total de unos $30 \mathrm{~mm}$, con una intensidad máxima cercana a los $4 \mathrm{~mm}$ en 10 minutos. El caudal máximo registrado fue de unos $600 \mathrm{l} / \mathrm{s}\left(125 \mathrm{l} / \mathrm{s} \cdot \mathrm{km}^{2}\right)$ y el caudal máximo fue 21 veces mayor que el caudal base; a su vez, se registraron una concentración máxima de sedimentos en suspensión de $764 \mathrm{mg} / \mathrm{l}$ y un total de sedimentos exportados de unos $2600 \mathrm{~kg}\left(542 \mathrm{~kg} / \mathrm{km}^{2}\right)$. En el gráfico de concentración se observan algunos picos anteriores al máximo que reproducen el mismo patrón que la intensidad de la precipitación, pero que sin embargo no aparecen claramente registrados en el hidrograma. 


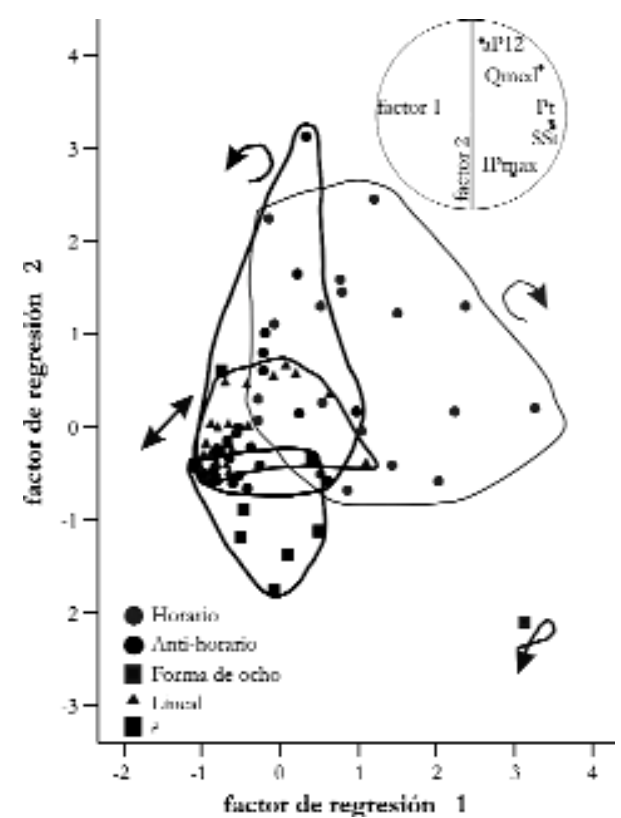

Figura 3. Distribución de los eventos de Aixola en el plano factorial I-II en relación con los tipos de bucles de histéresis.

Figure 3. Distribution of the events registered in Aixola in I-II factorial plane according to hysteretic loops.

Para este tipo de casos, BRASINGTON \& RICHARDS (2000) sugirieron que los sedimentos provienen sobre todo del lavado de las vertientes más que de la erosión riparia o del cauce. En cuanto al descenso de la concentración, éste es mucho más rápido que el del caudal. También en el gráfico CSS-Q se observa un descenso muy rápido de la concentración para los caudales más altos. Según WILLIAMS (1989), en estos casos de bucles horarios, ocurre un rápido agotamiento del sedimento antes de que el caudal llegue a su máximo. La curvatura hacia arriba presenta en los valores más altos del gráfico CSS-Q viene a reforzar esta misma idea del agotamiento del sedimento disponible, ya que, como se ha mencionado, aunque el caudal tiene una bajada muy lenta la concentración desciende rápidamente.

Los eventos con bucles en sentido anti-horario se ubican, en general, en la parte negativa o en el centro del factor I y tienden a dispersarse desde el centro hacia la parte positiva del factor II, por lo que son eventos de menores precipitaciones totales, caudales medios y exportación de sedimentos, y que, 

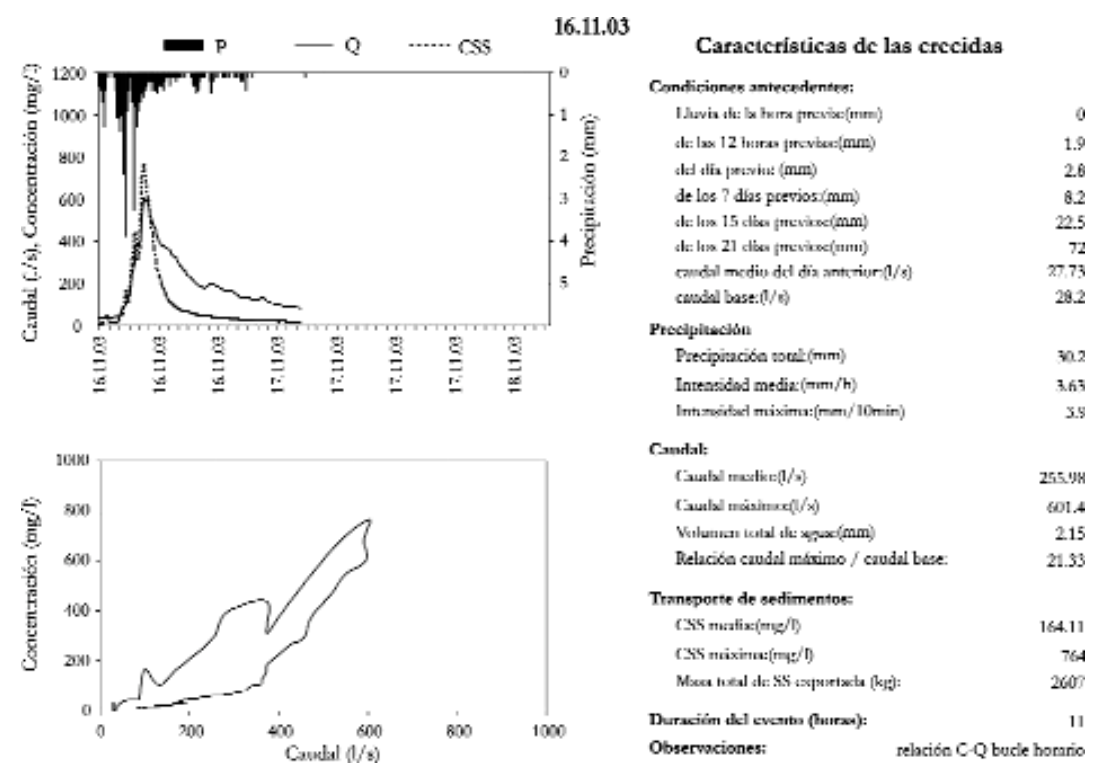

Figura 4. Representación gráfica del evento registrado el 16 de noviembre de 2003 en la estación de aforos de Aixola en el que se observa un bucle de histéresis horario entre la CSS y el caudal. En la figura superior se muestran el hidrograma, el sedimentograma y la precipitación del evento y en la inferior las relaciones CSS-Q durante el evento.

Figure 4. Graphic representation of the event registered during the 16th of November of 2003 in Aixola gauging station in which a clockwise hysteretic loop is observed. In the upper figure the hydrograph, the sedimentgraph and the precipitation of the event are shown and in the upper one CSS-Q relationship is drawn.

además, no presentan grandes intensidades de la precipitación y se pueden dar tanto bajo condiciones iniciales húmedas como más secas. En este tipo de eventos el pico de concentración es posterior al de caudal y, por lo tanto, el valor de la concentración en la rama ascendente del hidrograma es menor que en la rama descendente. Son varios los ejemplos que pueden ayudar a explicar este tipo de eventos, entre los cuales se describe a continuación el del 14 de abril de 2005 (Fig. 5). En este caso, la precipitación total registrada fue de $11.4 \mathrm{~mm}$, con una intensidad máxima de $1.9 \mathrm{~mm}$ en 10 minutos. El caudal máximo, de $441 \mathrm{l} / \mathrm{s}\left(92 \mathrm{l} / \mathrm{s} \cdot \mathrm{km}^{2}\right)$, fue 5 veces mayor que el caudal base y la masa total de sedimentos en suspensión transportada de unas 3 t (625 $\mathrm{kg} / \mathrm{km}^{2}$ ), con una concentración máxima registrada de $1123 \mathrm{mg} / 1$. El gráfico de concentración es más alargado que el hidrograma, aunque ambos son bastante estrechos, por lo que en el gráfico CSS-Q se observa un bucle anti- 
horario estrecho en el eje horizontal y alargado en el eje vertical. Este tipo de bucles se han explicado mediante la presencia de una fuente de sedimento significativa distante de la zona de mayor generación de escorrentía, o mediante la diferencia de la velocidad a la que viaja la onda de la crecida y la velocidad media del flujo que transporta la mayoría del sedimento en suspensión (WILLIAMS, 1989; BRASINGTON \& RICHARDS, 2000; SEEGER et al, 2004).
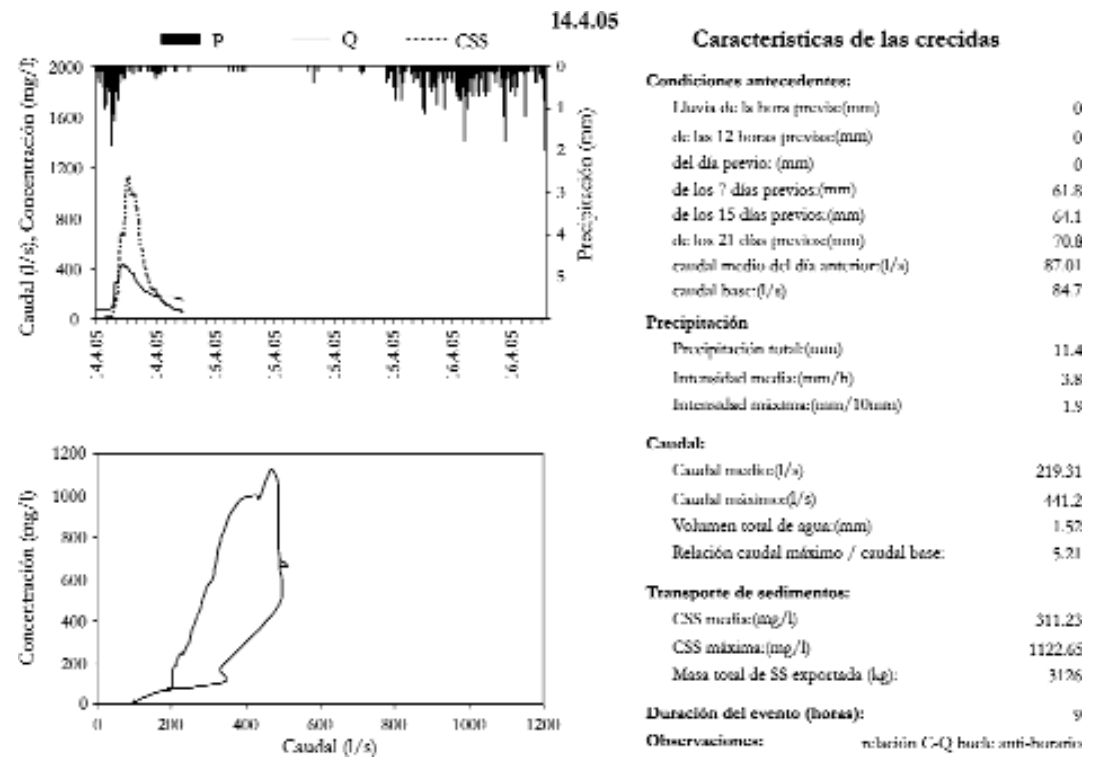

Figura 5. Representación gráfica del evento registrado el 14 de abril de 2005 en la estación de aforos de Aixola en el que se observa un bucle de histéresis anti-horario entre la CSS y el caudal. En la figura superior se muestran el hidrograma, el sedimentograma y la precipitación del evento y en la inferior las relaciones CSS-Q durante el evento.

Figure 5. Graphic representation of the event registered during the 14th of April of 2005 in Aixola gauging station in which a counter-clockwise hysteretic loop is observed. In the upper figure the hydrograph, the sedimentgraph and the precipitation of the event are shown and in the upper one CSS-Q relationship is drawn.

Los eventos que muestran una relación lineal entre CSS y Q se concentran en el centro del gráfico factorial, por lo que son eventos con bajas precipitaciones, caudales y volúmenes de exportación y también bajas intensidades de la precipitación. En la figura 6 se muestra uno de los eventos en los que las relaciones entre CSS y Q se pueden considerar como lineales 
dado que las diferencias existentes en la concentración de sedimentos entre la rama ascendente y la descendente del hidrograma son insignificantes. En la crecida del 30 de noviembre de 2003 (Fig. 6), por ejemplo, se registraron unos $12 \mathrm{~mm}$ de precipitación total con intensidades máximas de la misma de 1.2 $\mathrm{mm} / 10 \mathrm{~min}$. Teniendo en cuenta los dos picos visibles en el hidrograma, el volumen total de agua exportado fue de unos $2 \mathrm{~mm}$, con un caudal máximo de 327 1/s, además, la cantidad de sedimentos en suspensión exportados fue baja, de tan solo $321 \mathrm{~kg}$. En la relación entre la concentración y el caudal de la figura inferior se observa que, para ambos picos, apenas existen diferencias entre las concentraciones de sedimentos entre la rama ascendente y la descendente del hidrograma. WOOD (1977; recogido de WILLIAMS, 1989) relacionó este tipo de eventos con un continuo suministro de sedimentos en suspensión durante toda la crecida.

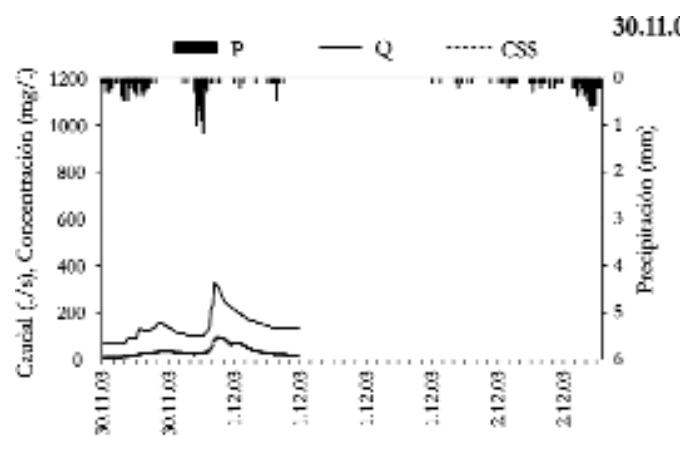

Cafactcristicas de las crccidas
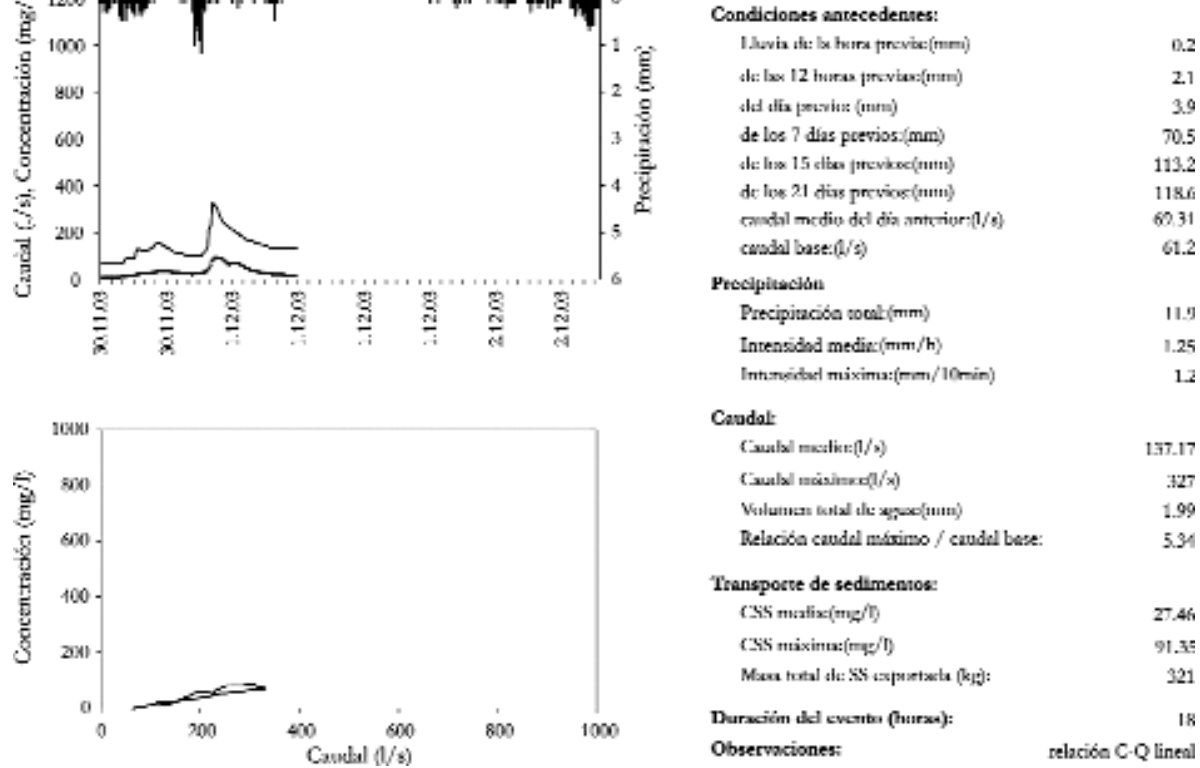

Observociones:

70.5

3.2

Figura 6. Representación gráfica del evento registrado el 30 de noviembre de 2003 en la estación de aforos de Aixola en el que se observa una relación lineal entre la CSS y el caudal. En la figura superior se muestran el hidrograma, el sedimentograma y la precipitación del evento y en la inferior las relaciones CSS-Q durante el evento.

Figure 6. Graphic representation of the event registered during the 30th of November of 2003 in Aixola gauging station in which a linear relationship is observed. In the upper figure the hydrograph, the sedimentgraph and the precipitation of the event are shown and in the upper one CSS-Q relationship is drawn. 
Otro tipo de relaciones concentración vs. caudal observadas, han sido las definidas por WILLIAMS (1989) como del tipo de bucles en forma de ocho (Fig. 7). Tal y como las definió Williams se ha observado un solo evento que se relaciona con altas intensidades máximas de la precipitación y condiciones antecedentes de poca humedad, ya que ocurrió en verano cuando las condiciones antecedentes son más secas. Son bucles que se pueden dividir en dos partes o sub-bucles que giran en direcciones opuestas; así, las relaciones CSS-Q son mayores para un rango de la rama ascendente del hidrograma y menores para otro rango, comparadas con los mismos valores de $\mathrm{Q}$ de la rama descendente del hidrograma. El evento del 9 de septiembre de 2004 muestra un bucle horario en la parte baja y uno anti-horario en la parte alta del gráfico CSS-Q. Este evento se genera a partir de una precipitación total de $33.1 \mathrm{~mm}$, con una intensidad máxima muy alta, de $6.6 \mathrm{~mm}$ en 10 minutos. Se registra así un caudal máximo de $1865 \mathrm{l} / \mathrm{s}\left(388 \mathrm{l} / \mathrm{s} \cdot \mathrm{km}^{2}\right)$, casi 73 veces mayor que el caudal base. La concentración máxima registrada en la estación de aforos fue de $8816 \mathrm{mg} / 1$ y se exportaron un total de $46 \mathrm{t}\left(9.6 \mathrm{t} / \mathrm{km}^{2}\right)$ de sedimentos en suspensión. Se trata, por lo tanto, de un evento de gran intensidad energética, aunque su duración, unas 15 horas, fuera bastante corta.

En la figura superior se observa que tanto el hidrograma como el gráfico de concentración son estrechos y puntiagudos y que ambos muestran un pequeño pico antes del pico principal, imitando al registro de la intensidad de la precipitación que también muestra dos máximos. Aunque la concentración de sedimentos en suspensión comienza a aumentar más rápidamente que el caudal, lo que parecía que se iba a convertir en un bucle de sentido horario, se convierte en la parte alta del gráfico CSS-Q en un bucle de sentido antihorario, debido a la mayor anchura del pico de concentración y a que cuando el caudal comienza a disminuir la concentración sigue aumentando. A partir del máximo, el agotamiento del sedimento disponible para ser exportado hace que la concentración de sedimentos disminuya rápidamente y el bucle anti-horario de la parte alta del gráfico se vuelva horario en la parte baja. SEEGER et al. (2004) relacionaron este segundo bucle anti-horario con momentos en los que las áreas de contribución se extienden a toda la cuenca debido a un flujo hortoniano generalizado que ocurre como consecuencia de las condiciones hidrológicas de la cuenca. Además, en la rama descendente del gráfico de concentración se observa un pico secundario, que no aparece en el hidrograma, y que, aunque al lado del pico principal parece insignificante, llega a alcanzar los $1000 \mathrm{mg} / 1$ de concentración, por lo que no es nada desdeñable. Este pico tardío podría estar relacionado con los picos de intensidad de la precipitación que se observan después de la crecida principal. 


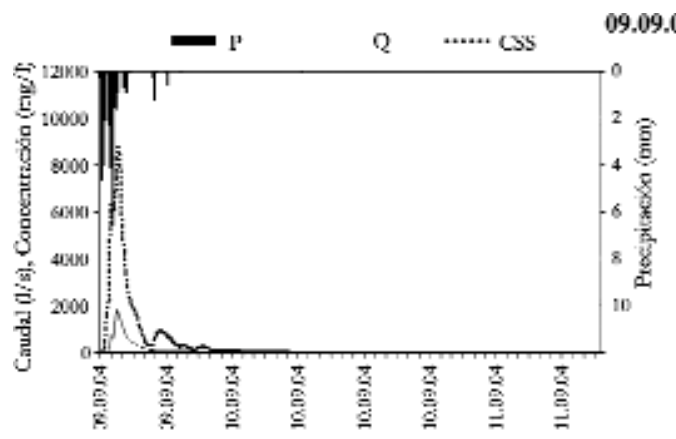

Característicasx de las crecidax
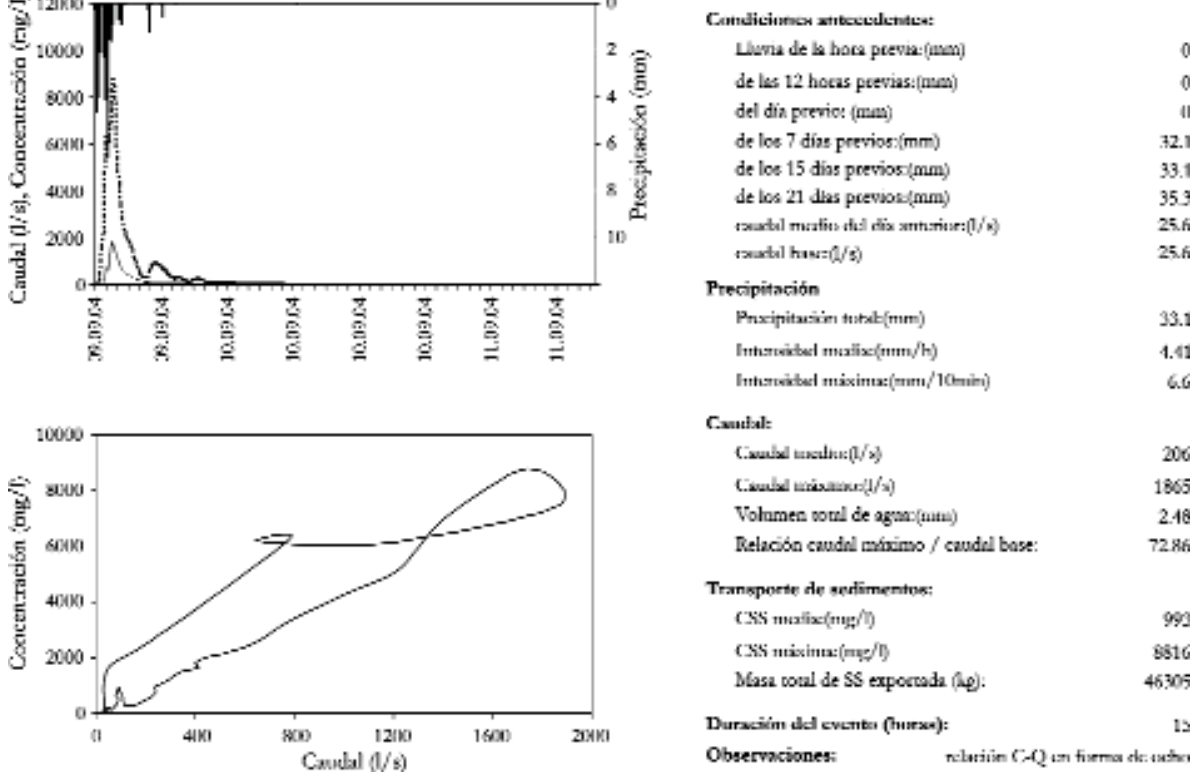

Figura 7. Representación gráfica del evento registrado el 9 de septiembre de 2004 en la estación de aforos de Aixola en el que se observa un bucle de histéresis en forma de ocho entre la CSS y el caudal. En la figura superior se muestran el hidrograma, el sedimentograma y la precipitación del evento y en la inferior las relaciones CSS-Q durante el evento.

Figure 7. Graphic representation of the event registered during the 9th of September of 2004 in Aixola gauging station in which an eight shaped hysteretic loop is observed. In the upper figure the hydrograph, the sedimentgraph and the precipitation of the event are shown and in the upper one CSS-Q relationship is drawn.

El resto de eventos, los que no han sido clasificados, también se dan en este tipo de condiciones de escasas precipitaciones antecedentes y altas intensidades de la precipitación causante del evento. Son eventos relacionados con las lluvias intensas ocurridas durante el verano. Por ejemplo, el evento del 20 de agosto de 2005 (Fig. 8) fue muy parecido al del 9 de septiembre de 2004 e incluso parece definir un bucle en forma de ocho, pero en realidad, y siendo fieles a la definición dada por Williams, no es así. Se trata de un evento en el que la precipitación total registrada fue de unos 23 $\mathrm{mm}$, con una intensidad máxima de $5.4 \mathrm{~mm}$ en 10 minutos. Estas precipitaciones provocaron caudales máximos de $1082 \mathrm{l} / \mathrm{s}\left(225 \mathrm{l} / \mathrm{s} \cdot \mathrm{km}^{2}\right)$, casi 35 veces mayores que el caudal base. Así, la concentración máxima de 
sedimentos en suspensión registrada, menor que en el caso anterior, es de $3028 \mathrm{mg} / 1$ con un total de sedimentos en suspensión exportados de 8 t (1.7 $\mathrm{t} / \mathrm{km}^{2}$ ). Pero en este caso, en el gráfico CSS-Q se observa un bucle de sentido horario en la parte más baja del mismo y otro muy tumbado, también de sentido horario, o incluso se podría decir que se trata de una relación casi lineal, en la parte alta. Así, parece que durante este evento, para caudales de entre 300 y 1082 l/s la disponibilidad de sedimentos para ser exportados es muy limitada.

Por último se ha examinado la evolución de la conductividad eléctrica en 28 de los eventos registrados en la estación de aforos en los que se han obtenido datos relativos a la conductividad eléctrica $(\mu \mathrm{S} / \mathrm{cm})$ de las muestras de agua recogidas por el tomamuestras automático (Fig. 9). Mediante estos
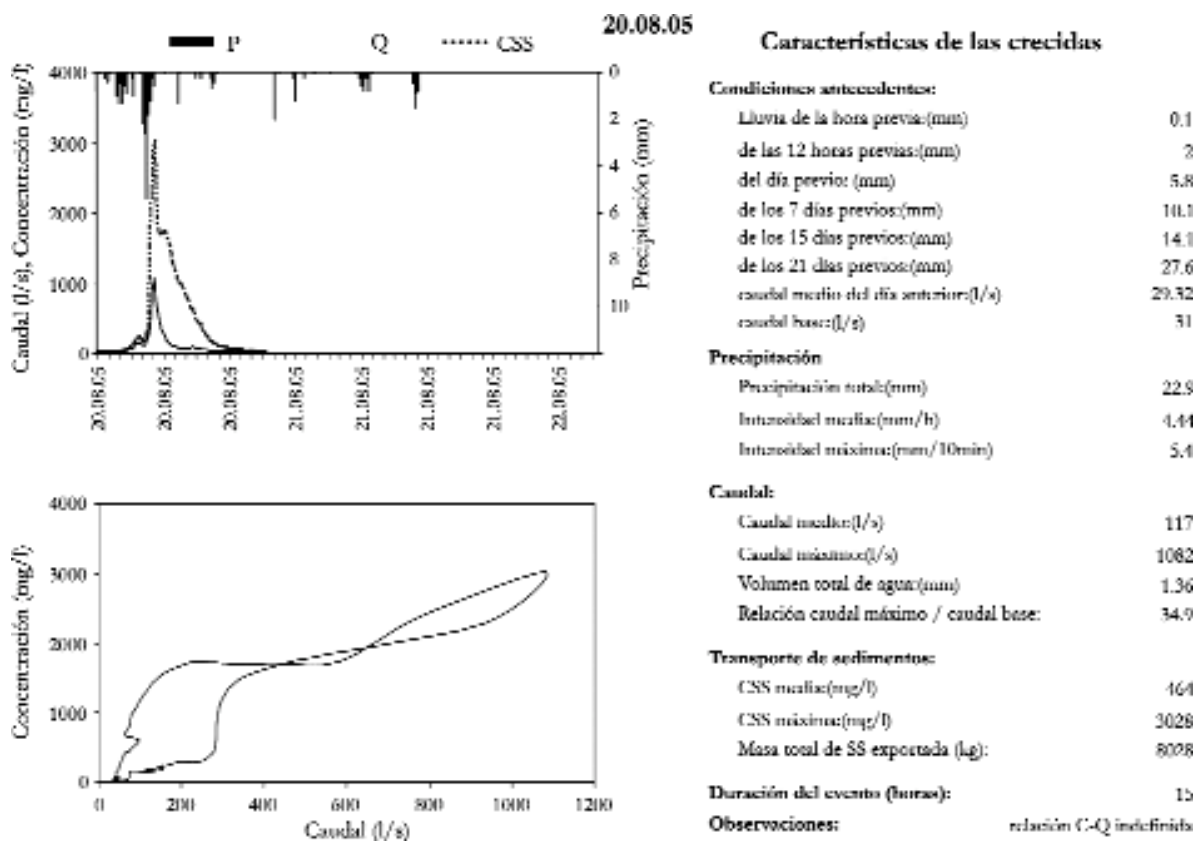

Obserwociones:

Figura 8. Representación gráfica del evento registrado el 20 de agosto de 2005 en la estación de aforos de Aixola en el que se observa un bucle de histéresis no definido entre la CSS y el caudal. En la figura superior se muestran el hidrograma, el sedimentograma y la precipitación del evento y en la inferior las relaciones CSS-Q durante el evento.

Figure 8. Graphic representation of the event registered during the 20th of August of 2005 in Aixola gauging station in which a non defined hysteretic loop is observed. In the upper figure the hydrograph, the sedimentgraph and the precipitation of the event are shown and in the upper one CSS-Q relationship is drawn. 
datos, ha sido posible observar un comportamiento bastante homogéneo de la calidad, en sentido de mineralización, de las aguas de la cuenca a lo largo de las diferentes situaciones hidrológicas. Se observa un descenso de la conductividad eléctrica con el aumento del caudal y una muy pronta recuperación de la conductividad después de cada evento. Además, parece existir un límite superior en la conductividad en torno a los $370 \mu \mathrm{S} / \mathrm{cm}$, lo que daría una idea de la conductividad de las aguas habitualmente presentes en la propia cuenca, consecuencia de su poder regulador (suelos, principalmente, debido a su espesor, y acuíferos). Pero además, llama la atención la existencia de otro límite, en este caso inferior, ya que, en la mayor parte de los eventos la conductividad no desciende por debajo de los 200 $\mu \mathrm{S} / \mathrm{cm}$, una conductividad, a pesar de todo, elevada, teniendo en cuenta que se trata de agua de mezcla (agua de lluvia $(\mathrm{CE} \approx 60-80 \mu \mathrm{S} / \mathrm{cm})+$ aguas del suelo y aguas subterráneas) lo cual induce a pensar que las aguas de la propia cuenca, subsuperficiales o subterráneas, están presentes en una gran proporción (40-45\%), incluso durante los picos de caudal, en la escorrentía total registrada en la estación de aforos. Sin embargo, aunque las aguas "previas" estén presentes en una gran proporción en el caudal total, la cuenca muestra una importante respuesta sedimentológica en la mayoría de los eventos registrados.

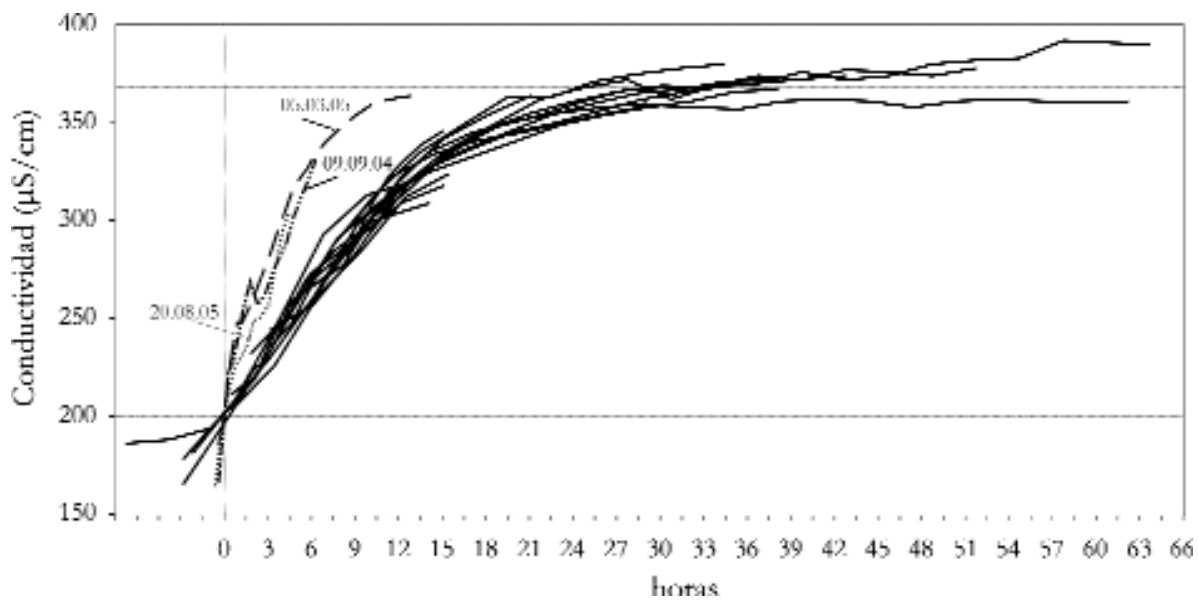

Figura 9. Evolución de la conductividad eléctrica $(\mu \mathrm{S} / \mathrm{cm}$, en horas) en las aguas del río Aixola después del pico máximo de caudal. $\mathrm{t}=0$ corresponde a una conductividad eléctrica de 200 $\mu \mathrm{S} / \mathrm{cm}$, seleccionada como un punto común de todos los eventos para facilitar la comparación entre ellos.

Figure 9. Evolution of electrical conductivity $(\mu \mathrm{S} / \mathrm{cm}$, in hours) in river waters after maximum discharge of runoff events registered in Aixola catchment. $t=0$ hours corresponds to $200 \mu \mathrm{S} / \mathrm{cm}$ electrical conductivity, selected as a common point in all the events in order to make comparison easier. 
En cuanto a la relación existente entre la conductividad de las aguas y su concentración de sedimentos en suspensión, se observa claramente que estas variables fluctúan en sentidos opuestos en función del caudal registrado. La figura 10 muestra la relación existente entre la conductividad eléctrica mínima y la concentración de sedimentos en suspensión máxima registrada durante cada crecida, ambos datos medidos en las muestras recogidas por el tomamuestras automático ubicado en la estación de aforos. Aunque no existe una clara relación de dependencia directa entre estos dos parámetros, se observa que las crecidas con menor conductividad, por debajo de los 190 $\mu \mathrm{S} / \mathrm{cm}$, más o menos, son las que registran mayores picos de concentración de sedimentos, en torno o por encima de $1000 \mathrm{mg} / \mathrm{l}$. Las crecidas del 9/9/2004, 20/8/2005 y 12/10/2005, son las que menores valores de conductividad muestran, con mínimos de 166, 163 y $161 \mu \mathrm{S} / \mathrm{cm}$ respectivamente. Las tres se sitúan en condiciones de baja humedad (finales de verano-principios de otoño) y presentan altas intensidades máximas de la precipitación, llegando hasta los $18.6 \mathrm{~mm}$ en diez minutos en el caso del

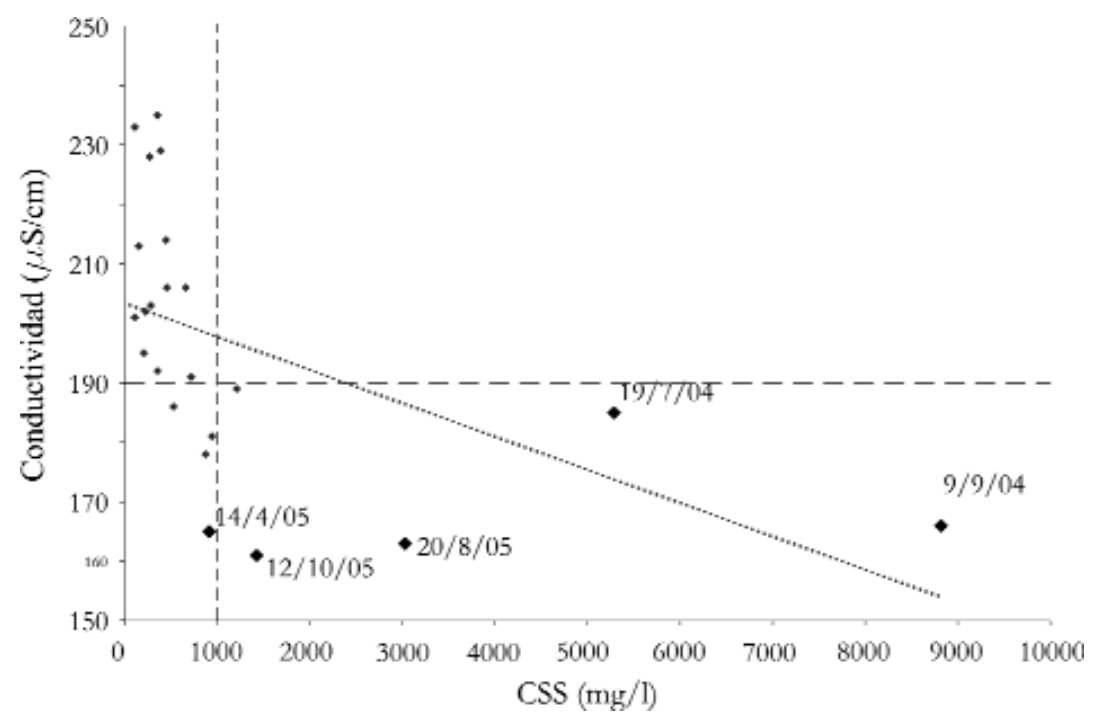

Figura 10. Relación entre la conductividad eléctrica mínima $(\mu \mathrm{S} / \mathrm{cm})$ y la concentración de sedimentos en suspensión máxima $(\mathrm{mg} / \mathrm{l})$ registrada durante cada crecida, ambos datos medidos en las muestras recogidas por el tomamuestras automático ubicado en la estación de aforos.

Figure 10. Relationship between minimum electrical conductivity $(\mu \mathrm{S} / \mathrm{cm})$ and maximum suspended sediment concentration $(\mathrm{mg} / \mathrm{l})$ registered during each event, both data were measured in the simples taken in the gauging station. 
evento del 12/10/2005, y registran además altos valores de CSS (mg/l), lo cual entra dentro de la lógica, ya que, la menor conductividad indica una mayor proporción de aguas provenientes de la escorrentía superficial con una mayor capacidad de arrastre de los sedimentos que se encuentran en las laderas, dando como resultado mayores concentraciones de sedimentos en suspensión.

\section{Conclusiones}

Teniendo en cuenta el conjunto de los eventos precipitación-escorrentía, existe una fuerte correlación entre los parámetros de precipitación, caudal y sedimentos en suspensión, pero no se observa una evidente correlación significativa entre éstos y las condiciones antecedentes al evento. Estos resultados sugieren una respuesta muy rápida de la cuenca a los eventos lluviosos, tanto en el caudal como en la exportación de los sedimentos, de modo que los eventos que se están analizando pueden ser considerados del tipo "flash flood". Además, no se han observado diferencias importantes en las relaciones entre parámetros obtenidos para cada año por separado, lo cual, también puede estar relacionado, al menos, con la rapidez de la respuesta que muestra la cuenca en las condiciones climáticas imperantes en el periodo analizado.

Mientras la exportación de sedimentos en suspensión durante un evento depende de la precipitación total registrada durante el mismo, la concentración de sedimentos se relaciona con la intensidad de la precipitación. Además, la correlación entre el caudal y la exportación de sedimentos es también importante y positiva. Sin embargo, los parámetros relacionados con los sedimentos en suspensión (exportación y concentración) no muestran ningún tipo de dependencia con respecto a las condiciones antecedentes al evento y la exportación de sedimentos ocurre sean cuales sean las condiciones hidrológicas imperantes en el momento en el que se registra un evento dado.

Basándose en las informaciones obtenidas sobre las relaciones entre $Q$ (caudal) y CSS durante las crecidas (bucles de histéresis), es posible definir diferentes procesos que están presentes en las cuencas a la hora de exportar los sedimentos. Se ha observado que dependiendo de las características de cada evento (caudal, precipitación y condiciones antecedentes) las relaciones entre el caudal y la concentración de sedimentos en suspensión durante los eventos son cambiantes, lo que sugiere una variabilidad espacial y temporal significativa en las fuentes de sedimento (JANSSON, 2002). 
Por otro lado, el análisis de la evolución de la conductividad eléctrica de las aguas durante las crecidas ha aportado información de interés, ya que es posible observar de una manera bastante precisa la dilución de las aguas, por mezcla con las aguas de precipitación durante los eventos, y su posterior recuperación en la decrecida. De sus relativamente altas conductividades mínimas y de su rápida recuperación se ha deducido que las aguas propias de la cuenca, las que llevan un tiempo en la misma, tienen una gran presencia durante todas las situaciones hidrológicas, también durante las crecidas, y ha quedado demostrada la importante inercia de esta cuenca. Además, se ha observado que en condiciones de caudales base bajos y de intensas precipitaciones, en Aixola se registran conductividades mínimas menores de lo habitual, relacionadas con una mayor generación de escorrentía superficial rápida y registros de concentración de sedimentos también mayores.

\section{Agradecimientos}

Los autores agradecen el apoyo del Departamento de Desarrollo Sostenible de la Diputación Foral de Gipuzkoa, la Universidad del País Vasco-Euskal Herriko Unibertsitatea y el Gobierno Vasco (Grupo IT-392).

\section{Referencias}

ALEXANDROV, Y., LARONNE, J.B. \& REID, I., 2003. Suspended sediment concentration and its variation with water discharge in a dryland ephemeral channel, northern Negev, Israel. Journal of Arid Environments, 53: 73-84.

ANKERS, C., WALLING, D.E. \& SMITH, R.P., 2003. The influence of catchment characteristics on suspended sediment properties. Hidrobiología, 494: 159-167.

BARTUREN, M.R. \& UGARTE, F.M., 1988. Evaluación de la carga detrítica en suspensión y carga en solución del río Oñati (Cuenca del río Deba. Gipuzkoa). Munibe, 40: 39-54.

BRASINGTON, J. \& RICHARDS, K., 2000. Turbidity and suspended sediment dynamics in small catchments in the Nepal Middle Hills. Hydrological Processes, 14: 2559-2574.

DE PABLO, C.T.F., DÍAZ PINEDA, F., MARTÍN DE AGAR, P. \& UGARTE, F.M., 1991. Pérdida de suelo y explotación forestal en el País Vasco. Bizia, 4: 35-38. 
EDESO, J.M., 1997. Pérdidas de suelo en explotaciones forestales de la vertiente cantábrica del País Vasco. En: Desarrollo rural y medio ambiente: el futuro del sector agrario. II jornadas de Urdaibai sobre desarrollo sostenible, 63-80.

FAO, UNESCO \& ISRIC, 1991. Mapa mundial de suelos, Leyenda revisada. Informe sobre el recurso mundial del suelo 60., Trad. por Carballas, Macías, Díaz, Carballas y Fernández. Sociedad Española de la Ciencia del Suelo, Santiago de Compostela, España.

FARNSWORTH, K.L. \& MILLIMAN, J.D., 2003. Effects of climatic and anthropogenic change on small mountainous rivers: the Salinas River example. Global and Planetary change, 39: 53-64.

GIPPEL, C.J., 1989. The use of turbidimeters in suspended sediment research. Hydrobiologia, 176/177: 465-480.

HOLEMAN, J.N., 1967. The sediment yield of major rivers of the World. Water Resources Research, 4 (4): 737-747.

HOUSE, W.A., LEACH, D., LONG, J.L.A., CRANWELL, P., BHARWAJ, L., MEHARG, A., RYLAND, G., ORR, D.O. \& WRIGHT, J., 1997. Microorganic compounds in the Humber rivers. Science of the Total Environment, 194/195: 357-373.

JANSSON, M.B., 2002. Determining sediment source areas in a tropical river basin, Costa Rica. Catena, 47: 63-84.

KNIGHTON, D., 1998. Fluvial forms and processes: a new perspective. Ed: Arnold, 386 p, London.

LAIGNEL, B., DUPUIS, E., DURAND, A., DUPONT, J.P. HAUCHARD, E. \& MASSEI, N., 2006. Erosion balance in the watersheds of the western Paris Basin by high frecuency monitoring of discharge and suspended sediment in surface water. C.R. Geoscience, 338: 556-564.

LENZI, M.A. \& MARCHI, L., 2000. Suspended sediment load during floods in a small stream of the Dolomites (northeastern Italy). Catena, 39: 267-282.

LEWIS, J., 1996. Turbidity-controlled suspended sediment sampling for runoff-event load estimation. Water Resources Research, 32(7): 2299-2310.

LLORENS, P., QUERALT, I., PLANA, F. \& GALLART, F., 1997. Studying solute and particulate sediment transfer in a small mediterranean mountainous catchment subject to land abandonment. Earth Surface Processes and Landforms, 22: 1027-1035.

MANEUX, E., DUMAS, J., CLEMENT, O., ETCHEBER, H., CHARRITTON, X., ETCHART, J., VEYSSY, E. \& RIMMELIN, P., 1999. Assessment of suspended matter input into the oceans by small mountainous coastal rivers: the case of the Bay of Biscay. Earth and Planetary Sciences, 329: 413420. 
MILLIMAN, J.D. \& MEADE, R.H., 1983. World wide delivery of river sediment to oceans. Journal of Geology, 91: 1-21.

MILLIMAN, J.D. \& SYVITSKI, J.P.M., 1992. Geomorphic/tectonic control of sediment discharge to the ocean: the importance of small mountainous rivers. Journal of Geology, 100: 525-544.

PICOUET, C., HINGRAY B. \& OLIVRY, J.C., 2001. Empirical and conceptual modelling of the suspended sediment dynamics in a large tropical African river: the Upper Niger river basin. Journal of Hydrology, 250: 19-39.

SCHUMM, S.A., 1977. The fluvial system. Wiley Interscience, 338 p, New York.

SEEGER M., ERREA M.-P., BEGUERÍA S., ARNÁEZ J., MARTÍ C. \& GARCÍA RUIZ J.M., 2004. Catchment soil moisture and rainfall characteristics as determinant factors for discharge/ suspended sediment hysteretic loops in a small headwater catchment in the Spanish Pyrenees. Journal of Hydrology, 288: 299-311.

SICHINGABULA, H.M., 1998. Factors controlling variations in suspended sediment concentration for single-valued sediment rating curves, Fraser River, British Columbia, Canada. Hydrological Processes, 12: 1869-1894.

SUN, H., CORNISH, P.S. \& DANIELL, T.M., 2001. Turbidity-based erosion estimation in a catchment in South Australia. Journal of Hydrology, 253: 227238.

URIARTE, A., 1998. Sediment dynamics on the inner continental shelf of the Basque Country (N. Spain). Department of Oceanography. Sciences Faculty. University of Southampton. Tesis Doctoral. $302 \mathrm{p}$.

WALLING, D.E., RUSSELL, M.A. \& WEBB, B.W., 2001. Controls on the nutrient content of suspended sediment transported by British Rivers. Science of the Total Environment, 266: 113-123.

WASS, P.D. \& LEEKS, J.L., 1999. Suspended sediment fluxes in the Humber catchment, UK Hydrological Processes, 13: 935-953.

WILLIAMS, G.P., 1989. Sediment concentration versus water discharge during single hydrologic events in rivers. Journal of Hydrology, 111: 89-106.

ZABALETA A., 2008. Análisis de la respuesta hidrosedimentaria en pequeñas cuencas de Gipuzkoa. Tesis Doctoral. UPV-EHU. Dpto. Geodinámica: 252 pp., anexos y CD.

ZABALETA A., MARTÍNEZ M., URIARTE J. \& ANTIGÜEDAD I., 2006. Sediment source variability as origin of uncertainties in sediment yield estimation (Aixola catchment, Basque Country). In: Pfister L., Matgen P., van den Bos R. and Hoffman L. (eds), Uncertainties in the 'monitoringconceptualization-modelling' sequence of catchment research. Abstract book of the ERB conference, Luxemburg: 27-28.

ZABALETA A., MARTÍNEZ M., URIARTE J. \& ANTIGÜEDAD I., 2007. Factors controlling suspended sediment yield during runoff events in small headwater catchments of the Basque Country. Catena, 71: 179-190. 